\title{
Salivary Melatonin Onset in Youth at Familial Risk for Bipolar Disorder
}

Neera Ghaziuddin $^{\mathrm{a}}$, Wael Shamseddeen ${ }^{\mathrm{a}}$, , Holli Bertram $^{\mathrm{a}}$, Melvin McInnis ${ }^{\mathrm{a}}$, Holly C. Wilcox ${ }^{\mathrm{c}}$, Philip B. Mitchell ${ }^{\mathrm{d}}$ Janice M. Fullerton $^{\mathrm{h}}$, Gloria M.P. Roberts ${ }^{\mathrm{d}}$, Anne L. Glowinski ${ }^{\mathrm{e}}$, Masoud Kamali $^{\mathrm{f}}$, Emma Stapp ${ }^{\mathrm{g}}$, Leslie A. Hulvershorn ${ }^{\mathrm{i}}$, Bipolar High Risk Research Group, John Nurnberger ${ }^{\mathrm{i}}$, Roseanne Armitage ${ }^{\mathrm{a}}$

${ }^{a}$ Department of Psychiatry, University of Michigan, Ann arbor, MI, USA

${ }^{\mathbf{b}}$ Department of Psychiatry, American University of Beirut, Lebanon

${ }^{\mathbf{c}}$ Johns Hopkins Schools of Public Health and Medicine, Baltimore, MD, USA

${ }^{\mathbf{d}}$ School of Psychiatry, University of New South Wales, Sydney, NSW, Australia \& Black Dog Institute, Prince of Wales Hospital, Sydney, NSW, Australia

${ }^{\mathrm{e}}$ Department of Psychiatry, Washington University School of Medicine, St. Louis, MO, USA

${ }^{\mathrm{f}}$ Department of Psychiatry, Massachusetts General Hospital, MA,USA " National Institute of Mental Health Intramural Research Program, Bethesda, MD, USA

${ }^{\mathrm{h}}$ Neuroscience Research Australia, Randwick, NSW, Australia \& School of Medical Sciences, University of New South Wales, Sydney, NSW, Australia

${ }^{\mathrm{i}}$ Department of Psychiatry, Indiana University School of Medicine, Indianapolis, IN, USA

Corresponding author: Neera Ghaziuddin, MD, MRCPsych

Conflict of interests for corresponding author: none

Phone: 734-764-0250

FAX: 734-936-8907

Acknowledgements: This study was funded by MICHR and the Richard Tam Foundation Word count: 177 (Abstract), 5447 (Text excluding abstract and the reference list)

This is the author's manuscript of the article published in final edited form as: 


\section{Highlights}

- The present study offers potential for identifying biological correlates (dysregulated sleep and melatonin onset) in adolescents who are at high risk for developing bipolar disorder due to a positive family history. While the current study involves a relatively small number of participants, it is likely to spur larger studies to identify potentially vulnerable youth for this disabling disorder.

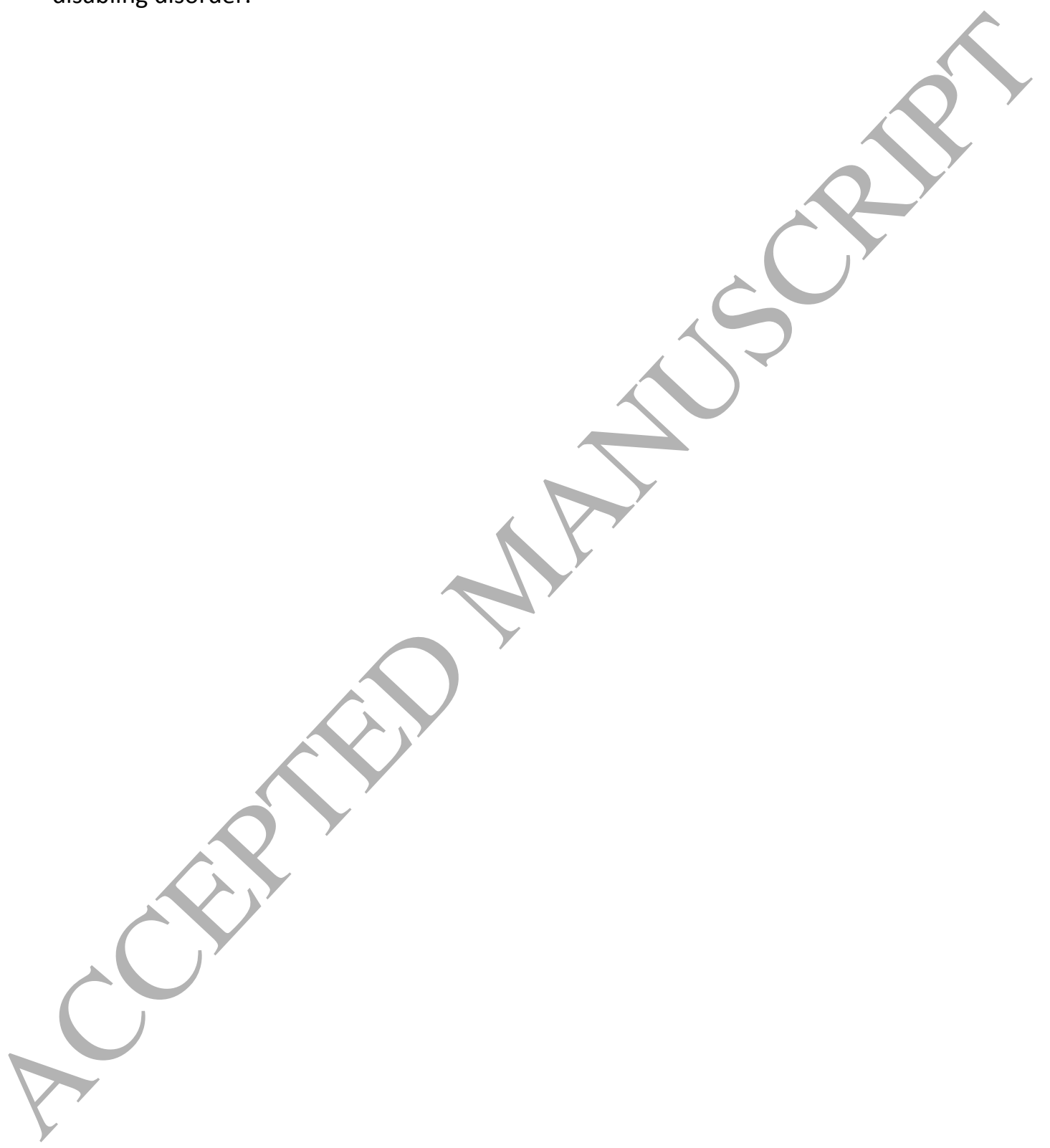




\begin{abstract}
Melatonin secretion and polysomnography (PSG) were compared among a group of healthy adolescents who were at high familial risk for bipolar disorder (HR) and a second group at low familial risk $(L R)$. Adolescent participants $(n=12)$ were a mean age $14 \pm 2.3$ years and included 8 females and 4 males. Saliva samples were collected under standardized condition light (red light) and following a 200 lux light exposure over two consecutive nights in a sleep laboratory. Red Light Melatonin onset (RLMO) was defined as saliva melatonin level exceeding the mean of the first 3 readings plus 2 standard deviations. Polysomnography was also completed during each night. HR youth, relative to LR, experienced a significantly earlier melatonin onset following 200 lux light exposure. Polysomnography revealed that LR youth, relative to HR, spent significantly more time in combined stages 3 and 4 (deep sleep) following red light exposure. Additionally, regardless of the group status (HR or LR), there was no significant difference in Red Light Melatonin Onset recorded at home or in the laboratory, implying its feasibility and reliability.
\end{abstract}

Key words: bipolar, risk, melatonin, polysomnography, adolescent 


\section{Introduction:}

Bipolar disorder (BP) is a serious and a disabling illness, associated with impairment in multiple domains of function. A positive family history of BP in a first degree relative (parent or a sibling) is a major risk factor for the later development of BP (Gershon et al. 1982; Tsuang and Faraone 1990). The rate for BP is $8-10 \%$ among children and adolescents who have one parent with BP, however, an overall increased rate of any type mood disorder is also observed in youth who have one parent with BP (5 to 67\%), when compared with offspring of healthy controls (0 to $38 \%$ ) (DelBello and Geller 2001).

A critically important challenge in clinical practice is to accurately identify children and adolescents who are currently symptom free but are at an elevated future risk due to their family history (Berk et al. 2007). It is believed that early detection and interyention among those at high risk for BP could be linked with the prevention of symptoms, reduced suicide risk, and improved course of illness, although studies are needed in this domain (Howes and Falkenberg 2011). These patients may show a range of non-specific symptoms including irritability, poor affect regulation, anxiety, depression, inattention, impulsivity, sleep disturbance, stubborn behaviors, unstable self-esteem, or rejection sensitivity (Chang et al. 2000; Shaw et al. 2005; Jones et al. 2006; Duffy et al. 2010). Disturbed sleep or anxiety may emerge as early as 3 years of age in those at familial risk for BP (Faedda et al. 2004). In fact, several studies have attempted to identify sleep and/or circadian disturbances in children at familial risk, using a variety of techniques such as sleep diaries, actigraphy, melatonin secretion and polysomnography (PSG). Results have varied depending on the technique used. For instance, using actigraphy in children of BP parents and controls, one study found greater subjective disturbance of sleep in children of BP parents (Jones et al. 2006). Ritter et al (2012) found that the sleep of high risk young adults (mean age $=25$ years) was more analogous to the sleep of BP patients, with both groups experiencing recurring episodes of insomnia or hypersomnia, difficulty in being able to shift circadian rhythms, difficulty in awakening, and episodes of prolonged sleep (Ritter et al. 2012).

Our interest in melatonin secretion and the effect of light in youth at risk for BP is based on published literature involving high risk young adults, euthymic adults with BPI, adult bipolar patients or those with other types of cyclical mood disorders and animal data. Specifically, the data show that i) melatonin is consistently reported as a reliable marker of circadian rhythms (Lewy 1983); ii) adolescents and young adults with no personal history of a major affective disorder but with parental BP (in one or both parents) were found to have a supersensitive suppressant response to light, suggesting that this may be a trait marker for BP (Nurnberger et al. 1988); iii) in medication-free euthymic BP 1 adults, melatonin levels following exposure to light fall twice as much compared to controls, underscoring the possibility that melatonin is a trait marker for BP (Lewy et al. 1985; Nurnberger et al. 2000); iv) acutely ill BP patients were noted to have lower baseline melatonin levels (Lam et al. 1990); v) involvement of melatonin has been postulated in cyclical mood disorders such as BP, cyclothymia and seasonal affective disorders (Pandi-Perumal et al. 2007); and vi) animal data provide additional support for the role of light in the pathophysiology of mood disorders (LeGates et al. 2012). LeGates et al (2012) had found 
that mice exposed to aberrant light cycles displayed increased depression-like behaviors and impaired learning (LeGates et al. 2012). Based on these observations, we hypothesized that melatonin secretion under dark and light conditions would significantly differ between adolescents at familial risk for BP, when compared to controls without a family history. We further hypothesized that salivary melatonin could be reliably measured at home in both participant groups.

Hormones most closely aligned with circadian rhythms, such as cortisol, melatonin or thyroid hormones have not been adequately studied in children and adolescents at familial risk. In humans, circadian rhythms are controlled by a "biological clock" in the suprachiasmatic nucleus ( $\mathrm{SCN}$ ), which is located in the anterior portion of the hypothalamus close to the optic nerve. The SCN is regulated by internal and external factors. Among external factors, also known as zeitgebers, light is one the most powerful influences. Abnormalities in circadian rhythms can include damped amplitude, a phase advance or delay, or a non 24 period (McClung 2007). These concepts apply to all measures of circadian rhythms, including the rest activity cycles, Red Light Melatonin Onset (RLMO) and melatonin secretion in response to a light exposure (LE).

The overall aims of the present study are to compare the effect of light on secretion of melatonin and polysomnography between high risk (HR) and low risk (LR) adolescents. HR adolescents were at an elevated risk for BP due to a positive family history of BP in a first degree relative (parent or a sibling). We chose to study melatonin secretion and sleep because these are objective phenomena that can be studied with little or no subjective input from adolescents.

Melatonin can be collected in youth in clinical practice and research, albeit with some training of the participants for accurately completing a timed-procedure. We also aimed to demonstrate that Red Light Melatonin Onset could be reliably measured in the home setting, in both groups, which could significantly reduce the cost of the procedure.

\section{Methods}

The investigators obtained IRB approval at the University of Michigan, Ann Arbor where this study was conducted.

\subsection{Subjects}

Participants were 14 youth (data are reported for 12) comprised of 10 females and 4 males (7 HR and $7 \mathrm{LR}$ ) ranging in age from 12 to 18 years. Subjects were recruited via flyers, advertisements and child and adolescent psychiatry clinics at the University of Michigan, Ann Arbor.

Participants were matched on age and gender but not on pubertal status and none were related to each other. A positive history of BP in a parent was determined based on history (from the parent), who had completed a Family Mental History form, and any other records provided by the parents. All HR participants had one parent with a positive history of BP, although one HR participant had both a parent and a sibling with the disorder. Presence of another mood disorder 
in first degree relatives, in addition to BP, was not an exclusion.

Exclusion criteria for all participants were DSM-IV diagnoses of BP, MDD, schizoaffective disorder, schizophrenia or substance abuse/dependence disorders; intellectual disability based on educational history; a significant medical or neurological disorder; and/or a history of taking any psychotropic medication during the previous week ( 3 weeks for fluoxetine) including antidepressants, antipsychotics, benzodiazepines, stimulant medication and/ or a non-stimulant agent for ADHD or a mood stabilizer.

Reported numbers vary based on participants who completed a particular procedure and/or based on the requirements of a given analysis (for instance, melatonin level $>3 \mathrm{pg} / \mathrm{ml}$ was not achieved by all participants). One subject was lost to contact prior to participation and one subject was missing some, but not all data (equipment malfunction when acquiring PSG and no saliva collected for LE). The total that completed all study procedures were 8 females $(H R=4)$ and 4 males $(H R=2)$, while partial data were available for one subject (belonged to LR group).

\subsection{Procedures}

Both subject groups participated in the collection of Red Light Melatonin onset (DLMO), melatonin secretion following 200 lux white light exposure (LE) over two hours, and polysomnography. These procedures were completed in the UM Sleep Laboratory over two consecutive nights. Standard red light melatonin onset protocol, described below, followed by sleep polysomnography and the light challenge night which included exposure to 200 lux light (during the first two hours of saliva collection) were completed by HR and LR groups. The order of the light challenge night was randomized. In the home setting, saliva collection under Red Light condition was limited to one night, was completed under identical conditions to Red Light Melatonin Onset in the laboratory and was completed by both groups.

The following standardized interviews and scales were completed at study-entry:

1. K-SADS-PL (Kiddie - Schedule for Affective Disorder and Schizophrenia - Present) (Kaufman et al. 1997): The screening portion of this semi-structured diagnostic interview was completed which is designed to assess current and past episodes of psychopathology in children and adolescents.

2. SCARED (Screen for Child Anxiety Related Emotional Disorders) (Birmaher et al. 1999): This is a child self-report instrument used to screen for childhood anxiety disorders.

3. CDI (Children's Depression Inventory) (Kovacs 1985): The participant completes this symptom-oriented scale to measure the severity of depressive symptoms.

4. CBQ-Adolescent (Conflict Behavior Questionnaire-Adolescent) (Robin and Foster 1989): This instrument is completed by the youth and is designed to assess the emotional quality of their interaction with each parent.

6. SEI (Self Esteem Index) (Brown and Alexander 1991): This is a self-completed instrument which measures self-perception and self-worth of the youth. 
7. HSS (Harter's Social Support Scale, Child) (Harter 1985): This scale has four different subscales including Parent Subscale, Classmate Subscale, Close Friend Subscale, and a Teacher Subscale.

8. FACES II (Family Adaptability and Cohesive Evaluation Scales) (Olson et al. 1991): This is a self-completed scale that determines how frequently a family participates in activities that are found to build cohesion and adaptability.

\subsection{Procedures during Red Light Melatonin Onset (RLMO)}

Based on their normal sleep patterns, for 5-7 days prior to their scheduled in-lab procedures, subjects kept sleep diaries and wore an actigraphy watch during the week prior to sleep study to verify adherence to the schedule. They were advised that a deviation in their bedtime or rise time, if greater than a half-hour, would result in exclusion from the study. Subjects arrived 5 hours before their scheduled bed-time to ensure adequate time to settle prior to the start of saliva collection and other procedures. For Red Light Melatonin Onset, they were given a pair of blueblocking, orange glasses upon their arrival and told to put them on. Though melatonin secretion is sensitive to light above 10 lux, the orange glasses block short wave length blue light (400-500 $\mathrm{nm}$ ) and provide a standardized environment (Middleton et al. 2002; Hanifin et al. 2006). The glasses also ensured that adolescents did not have to stay in very low light for the collection of Red Light Onset samples. Beginning 4 hours prior to their scheduled bed-time, saliva samples ( 9 for each subject) were collected every 30 minutes using Salivette tubes (Sarstedt, USA). The last saliva sample was collected at their stated bedtime, therefore, subjects did not remain awake beyond their bedtime. Based on this, in the statistical analyses, the bedtime was considered as the reference point, i.e. time " 0 ".

Fifteen minutes prior to collection of each saliva specimen, subjects brushed their teeth without toothpaste and rinsed their mouth. Subjects were also asked to remain seated for 5 minutes prior to each collection period, to minimize postural effects (Crowley et al. 2006). Each sample was collected by asking the subject to open the salivette tube into his or her mouth without touching the cotton and to roll the cotton around in their mouth for 2 minutes without chewing it. Then the subject was instructed to expectorate the cotton back into the salivette without touching it. They followed identical directions in the laboratory and their home, with the exception that during the laboratory collection, the staff assisted the subjects with timing themselves while they rolled the cotton in their mouth for 2 minutes. Samples were labeled, centrifuged within 2 hours of collection at $3000 \mathrm{rpm}$ (only lab-collected samples were centrifuges within 2 hours; homecollected samples were centrifuged the following day) for 10 minutes at room temperature and refrigerated at $-20^{\circ} \mathrm{C}$ prior to assay.

\subsection{Procedures for measuring melatonin secretion following exposure to 200 lux light (LE)}

Subjects underwent the LE protocol on one of two nights in the laboratory. Identical procedures described under Red Light Melatonin Onset were followed for the collection of saliva 4 hours prior to the subject's bedtime. The only addition during the LE protocol was exposure to 200 lux light for 2 hours that was started at the beginning of the 4-hour saliva collection period (subjects 
did not wear orange glasses during the light exposure). Standardization was achieved by instructing subjects to not look directly into the light source, watching a video of their choice during this two-hour light exposure and directing them to keep their eyes on the screen of a laptop computer placed several feet away to reduce the chance that they might look directly at the light.

\subsection{Procedure for home Red Light Melatonin Onset}

For home Red Light Melatonin Onset, subjects wore the orange-lensed glasses starting 4 hours prior to bedtime. The orange lenses attenuate the melatonin suppressing effects of light (Sasseville et al. 2006). Salivary samples were collected every $30 \mathrm{~min}$, following the same procedures as the laboratory Red Light Melatonin Onset, except that samples were labeled and refrigerated until they were collected by staff the following morning. Studies by Jensen et al. (2011) (Jensen et al. 2011) have shown the stability of salivary melatonin at room temperature. Based on this literature, we have conducted several experiments leaving salivary samples unrefrigerated for one week and freezing and thawing samples to demonstrate that melatonin assays would not be compromised during home collection.

Melatonin was assayed at the University of Michigan Core Assay Facility in the Department of Psychology, following published procedures (Conroy et al. 2012), but with age-appropriate melatonin onset criteria (Crowley et al. 2006). Samples containing $1 \mathrm{~mL}$ of saliva were assayed in duplicate for melatonin using a radioimmunoassay kit (American Laboratory Products, Windham, NH). The range of possible values is between .5 and $50 \mathrm{pg} / \mathrm{mL}$. The sensitivity of the assay is $0.2 \mathrm{pg} / \mathrm{mL}$, and the interassay variability ranges from $16 \%$ to $22 \%$ for a high concentration and from $1.7 \%$ to $2.4 \%$ for a low concentration.

\subsection{Polysomnography}

Polysomnography data were completed on both nights in the laboratory using a Vitaport III recording system, following standardized laboratory procedures. EEG was recorded from $\mathrm{C} 3$ and $\mathrm{C} 4$ electrodes, referenced to the earlobes and connected to a 10-k resistor to minimize nonhomogeneous current flow. The electrode montage also included left and right electrooculogram (EOG) leads placed on both the upper and the lower canthi; a bipolar, chin-cheek electromyography (EMG); leg leads, chest and abdomen respiration bands, and a nasal-oral thermistor. Impedances were maintained below $2 \mathrm{kOhms}$. The scoring of sleep was performed in accordance with the recommended standards and specification as outlined in the American Academy of sleep Medicine Manual (AASM) for the Scoring of sleep and Associated Events 2.0 (Berry et al. 2012). Scoring of respiratory events followed the AASM guidelines (Iber et al. 2007). Using adult criteria, where apneas were defined as $>90 \%$ reduction in air flow of at least $10 \mathrm{sec}$ duration and hypopneas were defined as a 30\% drop in flow of at least $10 \mathrm{sec}$ duration, with $\geq$ four percent desaturation; none of the subjects in the present study had an apnea hypopnea index $>0$. In fact, none of the participants showed respiratory events that lasted 20 seconds or longer and were associated with $\geq 3 \%$ desaturation. EEG arousal was defined as an abrupt shift 
to higher frequency of $>$ three seconds duration and preceded by $>10$ seconds of stable sleep (Scholle et al. 2012).

PSG variables included total sleep time, time in bed, sleep latency (to the first epoch of any sleep stage), REM latency, \% N1 (Stage 1), N2 (Stage 2) and N3 (Stages 3+ 4), \% REM, and wake time after sleep onset. Sleep efficiency was computed as the $\%$ total sleep time relative to time in bed.

\subsection{Analyses}

SPSS was used to conduct statistical analysis. Statistical significance was set at $p$-values less than 0.05 . Continuous variables (e.g. age) are presented as mean \pm standard deviation while categorical variables (e.g. gender) are presented as frequencies. Independent sample t-tests were used to compare LR and HR groups with respect to continuous variables, while Chi-square (or Fisher exact tests if the count was less than 5 in at least one cell) were used to test for associations between the two groups for categorical variables.

Generalized estimating equations (GEE) were used to compare the two groups with respect to melatonin onset. GEE models are an extension of generalized linear models in that they allow adjusting for correlations between observations (i.e. the correlation between melatonin onset under the red light and 200 lux light exposure for each participant) (Ziegler and Vens 2010). A GEE model was computed for each melatonin onset method, and the model included melatonin onset as the dependent variable and the risk group (HR compared to LR) and light condition (200 Lux compared to red light) as independent variables. If either variable was found to be significant, an interaction variable (i.e. risk group x light condition) was added to the model.

Similarly, two GEE models (one model for each melatonin onset method) were used to examine whether there was a difference between melatonin levels collected at home versus in the laboratory. Each model was conducted with melatonin onset as a dependent variable, location (home versus laboratory) as an independent variable and was then repeated while controlling for risk status (HR versus $\mathrm{LR}$ ).

Calculation of Melatonin onset in response to light exposure and Red Light Melatonin Onset was computed as follows: Red Light Melatonin Onset (RLMO) refers to the time when melatonin levels reach a certain threshold value, depending on the definition used. This was calculated using two different methods that are established in the literature and because there is a lack of consensus about the most optimum method (Lewy et al. 1999; Benloucif et al. 2005). The first method uses a $3 \mathrm{pg} / \mathrm{mL}$ as the threshold for salivary melatonin, while the second method is based on when the mean value derived from the first 3 samples exceeds 2 standard deviations (Voultsios et al. 1997; Conroy et al. 2012). Since participants were instructed to self-identify their bedtime and start the saliva collection 4 hours prior, bedtime was used as the reference time-point for each and was designated as time "0". Melatonin onset (under light exposure) and Red Light Melatonin Onset were designated as the earliest time before each participant's bedtime when they achieved the defined threshold level for melatonin. 
For the analyses involving the PSG data, we excluded an outlier with an exceedingly short total sleep duration of 25 minutes and another subject who had data only for one night.

\section{Results}

There was no significant age difference between HR and LR groups $(14.5 \pm 2.4$ vs. $14.5 \pm 2.3, p$ value $=1.00$ ). Comparison of standardized rating scales, which were completed only at baseline, revealed that the HR subjects had significantly greater affective lability scores $(11.7 \pm 6.2 \mathrm{vs}$. $5.3 \pm 4.4, p=0.04$ ) and lower adaptability and cohesion scores on the Family Adaptability and Cohesion scale $(80 \pm 19.4$ vs $101 \pm 8.3, p=04)$. There were no significant differences on other standardized scales (Table 1).

-Table 1 -

\subsection{Findings Related to Melatonin}

We examined melatonin secretion in three different ways. First, we examined a simple peak level; second, the time when a $3 \mathrm{pg} / \mathrm{mL}$ peak was reached; and third, when the melatonin level was 2 standard deviations above the mean of the first 3 measurements (Mean +2 SD) among LR and HR under red light or 200 Lux exposure. See figures 1-4.

There was no significant difference between the LR and HR groups with respect to peak melatonin level measured during 200 lux exposure $(9.8 \pm 6.1$ vs. $8.7 \pm 5.0, p$-value $=0.95$, Cohen $\mathrm{d}=0.2)$ or red light exposure $(12.5 \pm 9.6$ vs. $10.4 \pm 5.4, p$-value $=0.68$, Cohen $\mathrm{d}=0.3$; Figure 1). Further, there was no significant difference between groups with respect to time of peak melatonin level measured at 200 lux exposure $(p$-value $=0.70)$, red light exposure $(p$-value $=0.70)$, or at home ( $p$-value $=0.84)$.

-Figure 1 here-

Using generalized estimating equations and based on a threshold of $3 \mathrm{pg} / \mathrm{ml}$, there was no between-group difference in melatonin onset $(\mathrm{B}=21.5, p=0.37)$; however, within-subjects, there was a delayed melatonin secretion onset (closer to bed time) following exposure to 200 Lux as compared to the red light exposure $(B=-34.42, p=0.002)$ (Figure 2). When an interaction between bipolar risk status (HR vs. LR) and light exposure was added to the model, the interaction was not found to be significant $(\mathrm{B}=12.06, p=0.59)$ indicating that impact of light on melatonin was similar among the high risk and low risk groups.

\section{-Figure 2 here-}

On examining the computation that was based on calculating the mean of the first three melatonin values plus two standard-deviations (M+2SD's), a significant between-group effect was found; HR subjects experienced earlier Red Light Melatonin Onset compared to LR (B = 
47.9, $p=0.004$; Figure 3a). However, there was no within-subject effect (i.e. no impact of light exposure, $\mathrm{B}=-9.5, p=0.57$; Figure $3 \mathrm{~b}$ ). When the interaction between bipolar risk status and light exposure was added, a significant difference $(B=62.4, p=0.02)$ was found, which indicated that the illness-risk status conferred a greater difference in onset of melatonin secretion during the 200 Lux light compared to the red light exposure (Figure 3a).

\section{-Figures 3a and 3b here-}

There was no significant difference in peak melatonin levels measured under Red Light Melatonin Onset in laboratory $(11.5 \pm 7.7)$ and home $(11.6 \pm 9.8$; mean of difference $\pm \mathrm{SD}=0.2$ $\pm 7.7, p=0.91$, Cohen $\mathrm{D}=0.03)$. Similar results were noted when the two groups were considered separately: $(12.5 \pm 9.6$ vs. $13.3 \pm 11.0$, mean of difference $\pm \mathrm{SD} \neq 1.0 \pm 9.4, p=0.78$, $\mathrm{n}=7$ for LR group; $10.4 \pm 5.5$ vs. $9.7 \pm 8.9$, mean of difference $\pm \mathrm{SD}=0.6 \pm 5.9, p=0.80, \mathrm{n}=$ 6) at each setting (Figures $4 a$ and $4 b$ ). There was no also impact of setting (i.e. home vs. lab) on Red Light Melatonin Onset whether it was calculated based on a threshold of $3 \mathrm{pg} / \mathrm{ml}$ (mean difference $\pm \mathrm{SD}=8.9 \pm 39.6, p=0.2$, Cohen $\mathrm{D}=1.4$ ) or on $\mathrm{M}+2 \mathrm{SD}$ derived from the first three melatonin measurements (mean difference $\pm \mathrm{SD}=5.4 \pm 57.1, p=0.76$, Cohen $\mathrm{D}=0.1$ ).

\section{-Figure $4 a$ and $4 b$ here-}

\subsection{Findings Related to Polysomnography}

The LR group had a significantly higher percentage of N3 sleep (Stages $3+4$ sleep) on the Red Light Melatonin Onset night compared with HR $(26.2 \pm 1.1$ vs. $18.7 \pm 5.5, p<0.05)$. LR group also spent significantly longer wake time after sleep onset $(41.2 \pm 6.4$ vs. $11.3 \pm 8.1, \mathrm{p}<0.05)$ There were no significant differences in other sleep PSG measures. On the 200-lux light exposure night, there was a trend for a longer REM latency and a lower REM percentage among the HR group (Table 2).

\section{Discussion}

Findings of the present study include: (1) HR participants experienced significantly earlier melatonin onset in comparison to LR controls following 200 lux light exposure (defining melatonin onset as the point at which melatonin reaches two standard deviations above the mean of the first three readings); however, there were no significant between-group differences when using a $3 \mathrm{pg} / \mathrm{ml}$ level to define melatonin onset or when comparing peak melatonin levels ; (2) LR 
youth, spent significantly more time in N3 sleep than the HR group on the Red Light Melatonin Onset night under standard red light condition, and (3) there was no significant difference in Red Light Melatonin Onset under the standard red light condition recorded at home or in the lab setting, regardless of the risk status of the groups (HR or LR). We also found that the LR group had a longer time awake after sleep onset under the red light condition. Our overall findings indicate that there are identifiable circadian rhythm changes in asymptomatic adolescents who are at familial risk for BP. We must acknowledge that our finding of an earlier melatonin onset among the HR group under the 200 lux exposure is in direction which is opposite of that reported by Nurnberger et al. 1988, where the participants were similar, although older, when compared to the participants included in the present study. Below, we attempt to explain our findings on the basis of study design differences, while recognizing that the exact long-term implication of our findings is currently unknown, and there may be additional biological reasons pertaining to our findings which are beyond the scope of this study.

There is a paucity of literature in regards with melatonin secretion under light conditions and/ or Red Light Melatonin Onset in individuals at risk for BP. Although, it is notable that young adolescents such as those included in the present study have not been previously studied. Furthermore, meaningful comparisons are difficult because of somewhat inconsistent findings in the published literature and study-design differences. Nevertheless, the overall findings indicate that melatonin dysregulation is associated both with HR status and with a BP clinical diagnosis. For instance, in one of the earliest studies among this sparse literature, Nurnberger et al (1988) studied HR youth (age range $=15$ to 25 years, mean age of participants with one BP parent $=$ 20.5 years; subjects with one manic-depressive parent $=18$, major affective disorder on both sides of the family $=7$, controls $=20$ ); HR, relative to controls, were more sensitive to a melatonin-suppressant effect of 500 lux light exposure between 1 and 2 AM (Nurnberger et al. 1988). In another study, Nurnberger and colleagues (2000) studied euthymic adults with BP ( $\mathrm{n}=$ $29)$, unipolar disorder $(n=25)$ and controls $(n=50)$. No group differences were detected following a 500 lux light exposure between 2 and 4 AM. However, euthymic BP adults, relative to controls, had a lower baseline and peak melatonin levels on the night of light exposure, and the peak was noted to occur later on in the night when they were exposed to red light (Nurnberger et al. 2000). In one other study involving adults with BP $(\mathrm{n}=8)$, unipolar disorder $(n=7)$ or controls $(n=15)$, Lam et. al. $(1990)$, in response to 500 lux exposure from 1 AM to 2.15 AM, found greater nocturnal melatonin suppression among controls relative to BP who were experiencing an acute illness episode; however, BP had a lower baseline melatonin while unipolar participants did not differ from controls (Lam et al. 1990). In contrast to the aforementioned studies, Whalley and colleagues (1991) failed to identify a differential melatonin suppressant effect of 500 lux light exposure between 2 and 4 AM administered to medicationfree euthymic adults with BP disorder and controls ( $\mathrm{n}=15$ in each group); in that study, both groups experienced similar melatonin suppression following the light exposure (Whalley et al. 1991). We should note that the timing of the light exposure among these previously published studies was considerably different from the present study. Therefore, comparisons are difficult given that the timing of the light exposure has been shown to exert an important influence on 
melatonin secretion (Lewy 1983; Lewy et al. 1985; McIntyre et al. 1989).

An additional important design difference which makes it difficult to compare present findings with the previous literature are the participant related differences. The present study is notable for younger participants; subjects lacked a psychiatric diagnosis at the time of their participation; we measured melatonin onset versus melatonin secretion; and unlike the previous studies, we utilized a lower-intensity light exposure, which was standardized using the bedtime of each participant. We note that unlike previous studies, we found earlier melatonin onset in HR subjects following exposure to light; this finding is in direction that is opposite to what has been reported in the past.

Based on our findings and in context of the past work, we hypothesize that various different phases of bipolar disorder (high risk status, acute illness and euthymic states during a confirmed diagnosis) may be associated with subtle, yet variable melatonin secretion patterns. Thus, dysregulated patterns of melatonin secretion may be both a trait and a state marker for the illness. Only larger studies, which include carefully, defined subjects and standardized light and dark protocols can provide confirmatory evidence.

In regard to the PSG/ sleep related finding in the present study, we identified that the LR group, relative to HR, had significantly higher percentage of combined Stages 3 and 4 (N3), but only following the red light exposure. Unexpectedly, we also found longer awake time after sleep onset among LR under the red light condition. However, the higher N3 in LR is not accompanied by other sleep measures that would indicate more efficient sleep. Importantly, findings of the present study should be considered preliminary and require replication in a larger sample to understand these in context of other reported PSG disturbances in this subgroup. Specifically, past findings have included fragmented sleep in adolescents diagnosed with BP (Mullin et al. 2011), recurring insomnia and hypersomnia along with prolonged sleep latency among HR adolescents (Ritter et al. 2012), and longer stage 1 but diminished stage 4 among adolescents who later developed BP upon longitudinal follow up (Rao et al. 2002). We speculate that the shorter N3 in HR could be a marker of more widespread sleep disturbances during full-blown mood episodes, while only a larger sample size may detect additional previously reported deficits.

Although home Red Light Melatonin Onset is thought to be less expensive, there is scant evidence to support whether Red Light Melatonin Onset measured in the home is reliable with laboratory measurements. One previous study involving healthy adults ranging in age from 21 to 62 years found that Red Light Melatonin Onset assessed in the home of the participants was significantly correlated with Red Light Melatonin Onset completed in the laboratory (Burgess et al. 2015). Therefore, our finding regarding the high correlation between home and laboratory based Red Light Melatonin Onset in HR and LR adolescents replicates the findings of Burgess et al. (2015) and implies its feasibility and reliability.

It must be noted that we chose to present the data prior to correction for multiple testing (though 
correction is added as a footnote to table 2), for two reasons: firstly, there is an inherent difficulty in defining the number of independent tests for a fair correction to be applied; and secondly, due to the preliminary nature of the study, reporting of all suggestive findings will be more informative for future larger studies in defining specific variables for targeted analysis. Additional study limitations include: i) reliance on history to determine BP illness in first-degree relatives (instead of completing a diagnostic interview); ii) participants were matched on age and gender but were not matched on pubertal status, menstrual phase or season; iii) we do not report the participant's bedtime-schedules and actigraphy prior to salivary melatonin collection; iv) the peak melatonin levels only reflect the highest level achieved during the 4 hour saliva collection period and are not based on the entire night; v) we did not control for ambient light; vi) the study is underpowered to check for interactions and, vii) due to the cross sectional nature of the study design, we do not yet know the longitudinal predictive value of these findings. Additionally, we acknowledge that it is beyond the scope of this paper to discuss the mechanisms underlying the association between melatonin and $\mathrm{BP}$

While it may be premature to draw definite conclusions, our findings indicate that Red Light Melatonin Onset and melatonin secretion following a 200-lux light exposure may be relatively inexpensive tools, which could be used either alone or along with the more expensive laboratorybased PSG, for characterizing the circadian rhythms of HR youth. Our findings indicate that the HR group, relative to the LR, displayed a greater disruption in melatonin secretion and in their sleep pattern. Red Light Melatonin Onset, using the red light condition, could be reliably utilized in either subject-group in the home setting, which addresses important feasibility issues, which have thus far limited the scale of similar studies. The implications of these findings can only be clarified by larger studies, which should ideally include longitudinal outcomes. Any reliable, inexpensive test with potential for predicting outcome could open the possibility for developing and implementing prevention strategies, which may improve outcome; this continues to be the overarching goal of this research.

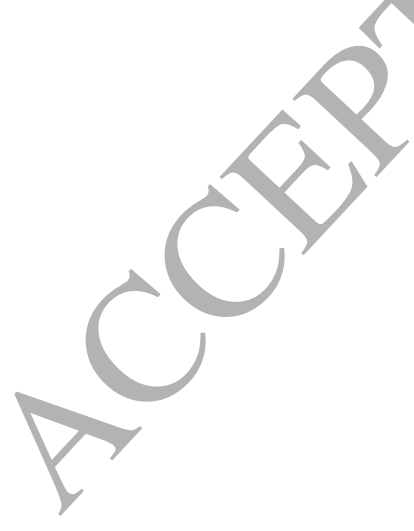




\section{References:}

Benloucif, S., Guico, M. J., Reid, K. J., Wolfe, L. F., L'Hermite-Baleriaux, M. and Zee, P.C. (2005). Stability of melatonin and temperature as circadian phase markers and their relation to sleep times in humans. J Biol Rhythms_20: 178-188.

Berk, M., Conus, P., Lucas, N., Hallam, K., Malhi, G. S., Dodd, S., Yatham, L. N., Yung, A., Mcgorry, P. (2007). Setting the stage: from prodrome to treatment resistance in bipolar disorder. Bipolar Disorders 9(7): 671-678.

Berry, R. B., Budhiraja, R., Gottlieb, D. J., Gozal, D., Iber, C., Kapur, V. K., Marcus, C. L., Mehra, R., Parthasarathy, S., Quan, S. F., Redline, S., Strohl, K. P., Davidson Ward S.L., Tangredi M. M. (2012). The AASM Manual for the scoring of Sleep and Associated Events: Rules Terminology and Technical Specifications, Version 2.0. American Academy of Sleep Medicine 2012. , Darien, IL., American Academy of Sleep Medicine.

Birmaher, B., Brent, D. A., Chiappetta, L., Bridge, J., Monga, S., Baugher, M. (1999). Psychometric properties of the Screen for Child Anxiety Related Emotional Disorders (SCARED): a replication study. Journal of the American Academy of Child \& Adolescent Psychiatry 38(10): 1230-1236. Brown, L. and A. Alexander (1991). Self esteem index., Psychological Assessment Resources, Inc. (PAR).

Burgess, H. J., Wyatt, J. K., Park, M., Fogg, L. F. (2015). Home circadian phase assessments with measures of compliance yield accurate dim light melatonin onsets. Sleep 38(6): 889-897.

Chang, K. D., Steiner, H., Ketter, T. A. (2000). Psychiatric phenomenology of child and adolescent bipolar offspring. Journal of the American Academy of Child \& Adolescent Psychiatry 39(4): 453460.

Conroy, D., Hairston, I., Arnedt, J. T., Hoffmann, R., Armitage, R., Brower, K. (2012). Dim light melatonin onset in alcohol-dependent men and women compared to healthy controls. Journal of Biological Rhythms 29(1):35-42.

Crowley, S. J., Acebo, C., Fallone, G., Carskadon, M. A. (2006). Estimating dim light melatonin onset (DLMO) phase in adolescents using summer or school-year sleep/wake schedules. Sleep 29: 16321641.

Delbello, M. P. and Geller, B. (2001). Review of studies of child and adolescent offspring of bipolar parents. Bipolar Disorders 3(6): 325-334.

Duffy, A., Alda, M., Hajek, T., Sherry, S. B., Grof, P. (2010). Early stages in the development of bipolar disorder. Journal of affective disorders 121(1-2): 127-135.

Faedda, G. L., Baldessarini, R. J., Glovinsky, I. P., Austin, N. B. (2004). Pediatric bipolar disorder: phenomenology and course of illness. Bipolar disorders 6(4): 305-313.

Gershon, E. S., Hamoit, V., Guroff, J. J. (1982). A family study of schizoaffective, bipolar I, bipolar II, unipolar and normal control probands. Arch Gen Psychiatry 39(10): 1157-1167.

Hanifin, J. P., Stewart, K. T., Smith, P., Tanner, R., Rollag, M., Brainard, G. C. (2006). High-intensity red light suppresses melatonin. Chronobiology International 23(1-2): 251-268.

Harter, S. (1985). Manual for the Social Support Scale for Children. Denver, University of Denver. Howes, O. D. and I. Falkenberg (2011). Early detection and intervention in bipolar affective disorder: targeting the development of the disorder. Current Psychiatry Rep 13(6): 493-499.

Iber, C.,Ancoli-Israel, S., Chesson, A., Quan, S. (2007). The AASM manual for the scoring of sleep and associated events; rules, terminology and technical specifications. . A. A. o. S. Medicine. Westchester, IL.

Jensen, M. A., Hansen, A. M., Abrahmsson, P., Norgaard, A. W. (2011). Development and evaluation of a liquid chromatography tandem mass spectometry method for simultaneous determination of salivary melatonin, cortisol and testosterone. J Chromatogr B Analyt Technol Biomed Life Sci 879(25): 2527-2532. 
Jones, S. H., Tai, S., Evershed, K., Knowles, R., Bentall, R. (2006). Early detection of bipolar disorder: a pilot familial high-risk study of parents with bipolar disorder and their adolescent children. Bipolar disorders 8(4): 362-372.

Kaufman, J., Birmaher, B., Brent, D., Rao, U., Flynn, C., Moreci, P., Williamson D., Ryan N. (1997). Schedule for Affective Disorders and Schizophrenia for School-Age Children-Present and Lifetime Version (K-SADS-PL): initial reliability and validity data.see comment. Journal of the American Academy of Child \& Adolescent Psychiatry 36(7): 980.

Kovacs, M. (1985). "The Children's Depression, Inventory (CDI)." Psychopharmacology bulletin 21(4): 995.

Lam, R. W., Berkowitz, A. L., Berga, S. L., Clark, C. M., Kripke, D. F., Gillin, J. C. (1990).Melatonin suppression in bipolar and unipolar mood disorders. Psychiatry Research 33(2): 129-134. Legates, T. A., Altimus, C. M., Wang, H., Lee, H. K., Yang, S., Zhao, H., Kirkwood, A., Weber, E. T., Hattar, S. (2012). Aberrant light directly impairs mood and learning through melanopsinexpressing neurons. Nature Medicine 491: 594-598.

Lewy, A. J. (1983). Effects of light on human melatonin production and the human circadian system. Progress in Neuro-Psychopharmacology \& Biological Psychiatry 7(4-6): 551-556.

Lewy, A. J., N. L. Cutler and R. L. Sack (1999). The endogenous melatonin profile as a marker for circadian phase position. J Biol Rhythms 14: 227-236.

Lewy, A. J., Nurnberger, J. I., Wehr, T. A., Pack, D., Becker, L. E., Powell, R. L., Newsome, D. A. (1985). Supersensitivity to light: possible trait marker for manic-depressive illness. American Journal of Psychiatry 142(6): 725-727.

Mcclung, C. A. (2007). Circadian genes, rhythms and the biology of mood disorders. Pharmacology \& Therapeutics 114(2): 222-232.

Mcintyre, I. M., Norman, T. R., Burrows, G. D., Armstrong, S. M. (1989). Human melatonin suppression by light is intensity dependent. Journal of Pineal Research 6(2): 149-156.

Middleton, B., Stone, B. M., Arendt, J. (2002). Human circadian phase in 12:12 h, 200: <8 lux and 1000: <8 lux light-dark cycles, without scheduled sleep or activity. Neuroscience Letters 329(1): 4144.

Mullin, B. C., Harvey, A. G., Hinshaw, S. P. (2011). A preliminary study of sleep in adolescents with bipolar disorder, ADHD and non-patient controls. Bipolar Disord 13(4): 425-432.

Nurnberger, J. I., Berrettini, W., Tamarkin, L., Hamovit, J., Norton, J., E. Gershon, E. (1988).

Supersensitivity to melatonin suppression by light in young people at high risk for affective disorder. A preliminary report. Neuropsychopharmacology 1(3): 217-223.

Nurnberger, J. I., Adkins, S., Lahiri, D. K. (2000). Melatonin suppression by light in euthymic bipolar and unipolar patients. Archives of General Psychiatry 57(6): 572-579.

Olson, D. H., Bell, R., Portner, J. (1991). FACES II. Family Social Science: 1-20.

Pandi-Perumal, S.R., Smits, M., Spence, W., Srinivasan, V., Cardinali, D.P., Lowe, A.D., Kayomov, L. (2007). Dim light melatonin onset (DLMO): a tool for the analysis of circadian phase in human sleep and chronobiological disorders. Progress in Neuro-Psychopharmacolgy and Biological Psychiatry 31(1):1-11.

Rao, U., Dahl, R. E., Ryan, N. D., Birmaher, B., Williamson, D.E., Rao, R., Kaufman, J. (2002). Heterogeneity in EEG sleep findings in adolescent depression: unipolar versus bipolar clinical course. Journal of affective disorders 70(3): 273-280.

Ritter, P. S., Marx, C., Lewtschenko, N., Pfeiffer, S., Leopold, K., Bauer, M., Pfennig, A. (2012). The characteristics of sleep in patients with manifest bipolar disorder, subjects at high risk of developing the disease and healthy controls. Biological Psychiatry 119(10): 1173-1184. Robin, A. L. and S. L. Foster (1989). Negotiating parent-adolescent conflict: a behavioral-family approach. New York., Guildfred Press. 
Sasseville, A., Pacquin, N., Sevigny, J., Herbert, M. (2006). Blue blocker glasses impede the capacity of blight light to suppress melatonin production. LPineal Res. 41(73-78.).

Scholle, S., Wiater, A., Scholle, H. (2012). Normative values of polysomnographic parameters in childhood and adolescence: Arousal events. Sleep Medicine 13: 243-251.

Shaw, J. A., Egeland, J. A, Endicott, J., Allen, C. R., Hostetter, A. M. (2005). A 10-year prospective study of prodromal patterns for bipolar disorder among Amish youth. Journal of the American Academy of Child \& Adolescent Psychiatry 44(11): 1104-1111.

Tsuang, M. T. and Faraone, S. V. (1990). The genetics of mood disorders. Baltimore, MD., US, Johns Hopkins University Press.

Voultsios, A., Kennaway, D. J., Dawson, D. (1997). Salivary melatonin as a circadian phase marker: validation and comparison to plasma melatonin. Journal of Biological Rhythms 12(5): 457-466. Whalley, L. J., Perini, T., Shering, A., Bennie, J. (1991). Melatonin response to bright light in recovered, drug-free, bipolar patients. Psychiatry Research 38(1): 13-19.

Ziegler, A. and Vens, M. (2010). Generalized esimating equations. Notes on the choice of the working correlation matrix. Methods Inf Med. 49(5): 421-432. 
Table 1: Clinical Factors among Low versus High Risk Groups

\begin{tabular}{lccc}
\hline & Low Risk Group & High Risk Group \\
& Mean \pm SD & Mean \pm SD & p-value \\
\hline Age & $14.5 \pm 2.3$ & $14.5 \pm 2.4$ & 1.00 \\
Gender (Female) & $\mathrm{N}=6 ;(75 \%)$ & $\mathrm{N}=4 ;(66.7 \%)$ & 1.00 \\
Children Affective Lability Scale & $5.3 \pm 4.4$ & $11.7 \pm 6.2$ & $0.04^{*}$ \\
Children's Behavior Questionnaire & & & \\
Father & $2.4 \pm 3.7$ & $3.8 \pm 3.8$ & 0.48 \\
Children's Behavior Questionnaire & & & \\
Mother & $1.1 \pm 1.6$ & $1.8 \pm 1.3$ & 0.39 \\
Children's Depression Inventory & $25.3 \pm 1.8$ & $23.3 \pm 2.3$ & 0.10 \\
Screen for Child Anxiety Related & & & \\
Disorders (SCARED) & $12.4 \pm 8$ & $15.2 \pm 9.0$ & 0.57 \\
Family Adaptability \& Cohesion Scale & $101 \pm 8.3$ & $80 \pm 19.4$ & $0.04 *$ \\
Harter Social Support Scale & $3.5 \pm 0.4$ & $3.2 \pm 0.3$ & 0.28 \\
Self Esteem Index & $390.4 \pm 337$ & $249.3 \pm 31.3$ & 0.33 \\
\hline \multicolumn{1}{c}{$p<0.05^{*}$} & & & \\
\hline
\end{tabular}


Figure 1: Peak Melatonin Level (pg/ml) by light exposure 25

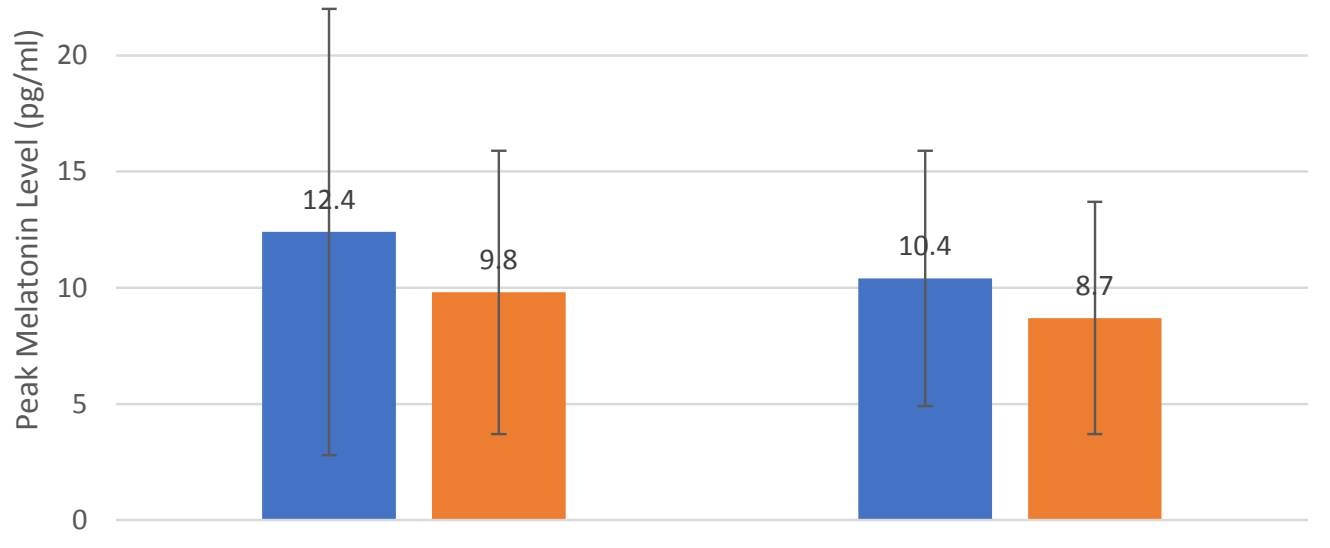

Low risk

High risk

Risk group stratified by light exposure

— Red light $\quad 200$ lux

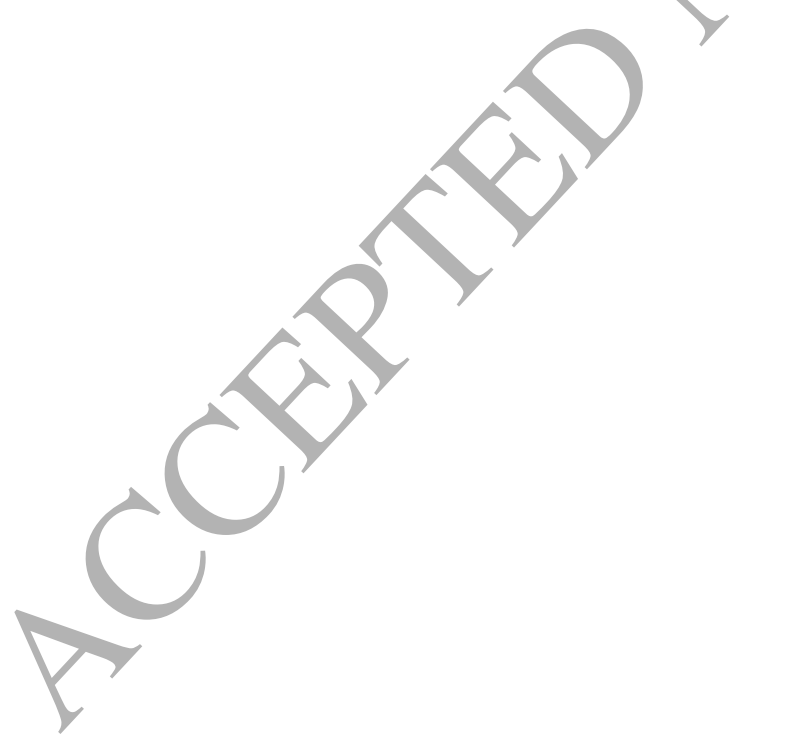


Figure 2: Melatonin Onset Following Red and 200 Lux Light (cutoff $>3$ pg/ml)

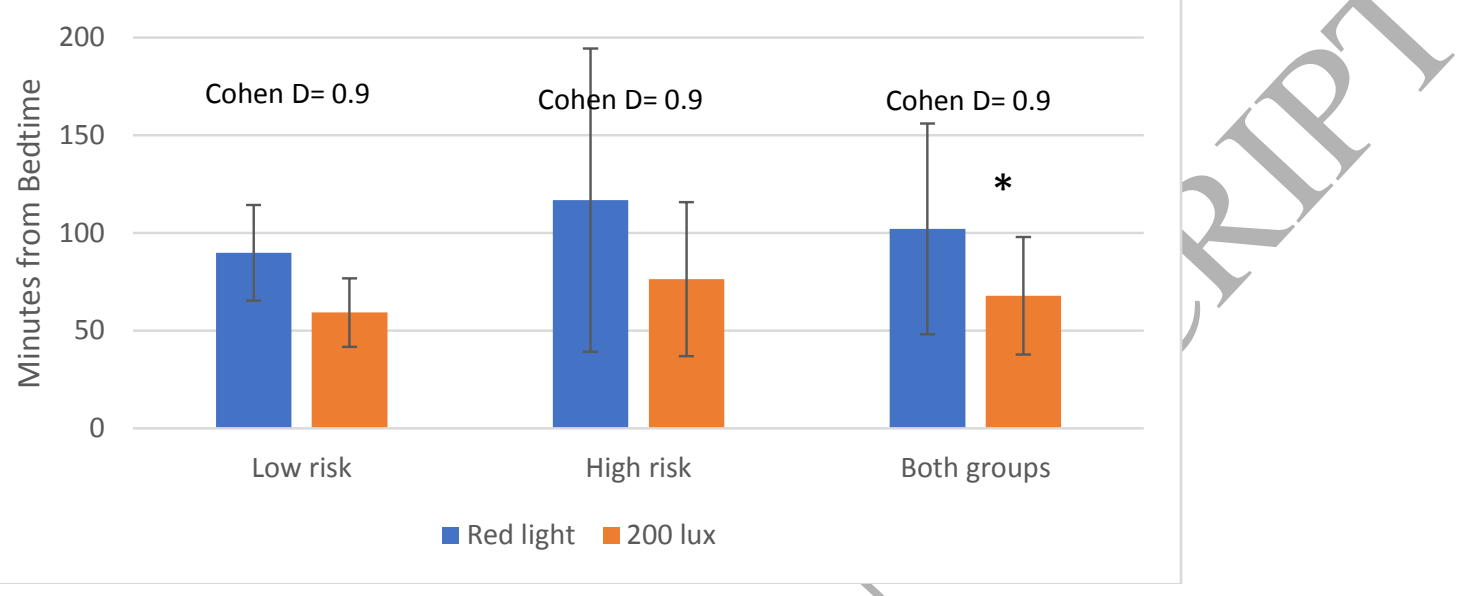


Figure 3: Melatonin Onset Following Red and 200 Lux Light Exposure (cutoff > M+2SD) a)Each Setting stratified by risk group

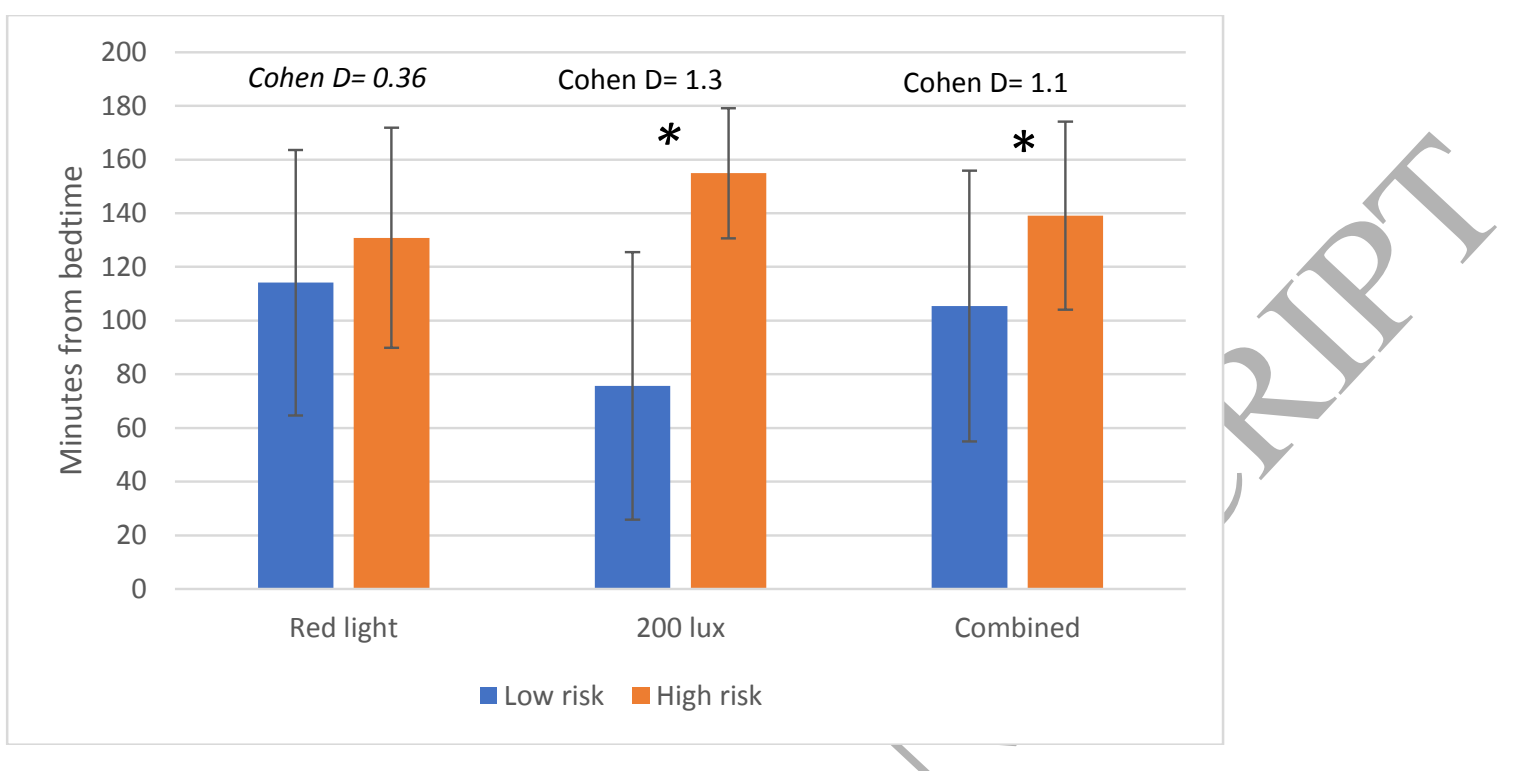

b) Each Risk group stratified by setting.

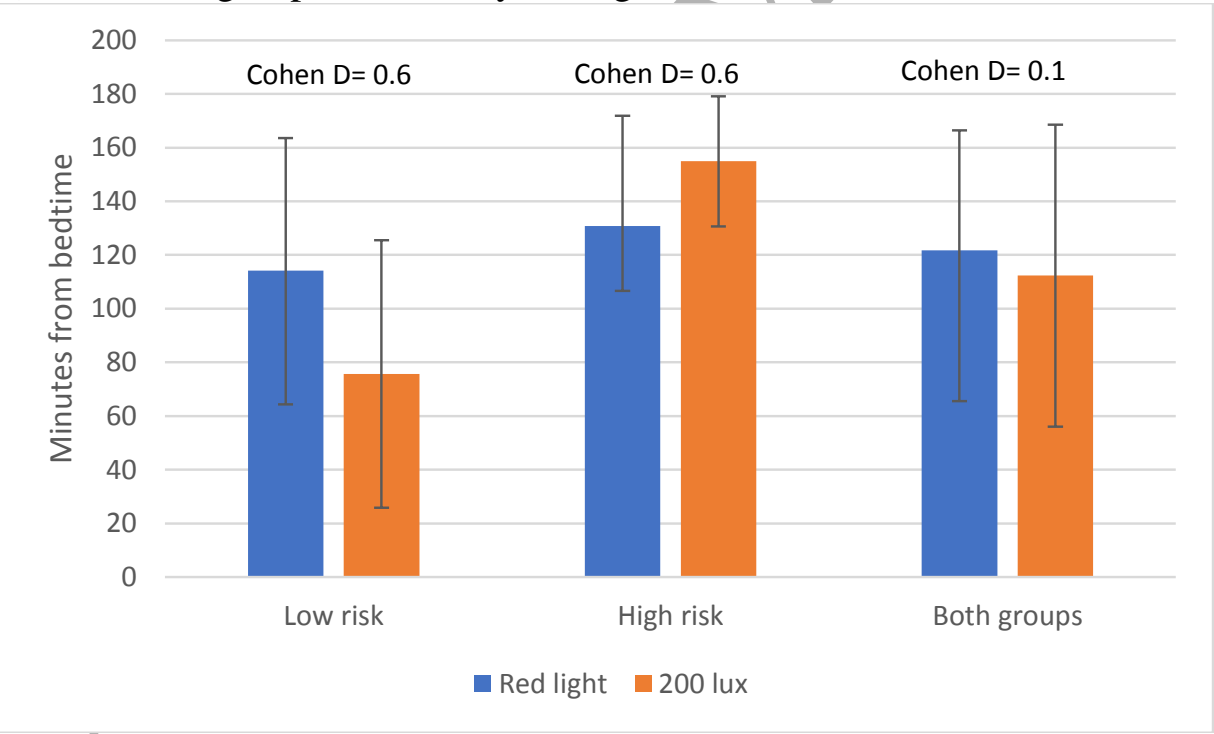


Figure 4: Melatonin Onset in Laboratory Compared to Home; using different cutoff points a) threshold of $3 \mathrm{pg} / \mathrm{ml}$
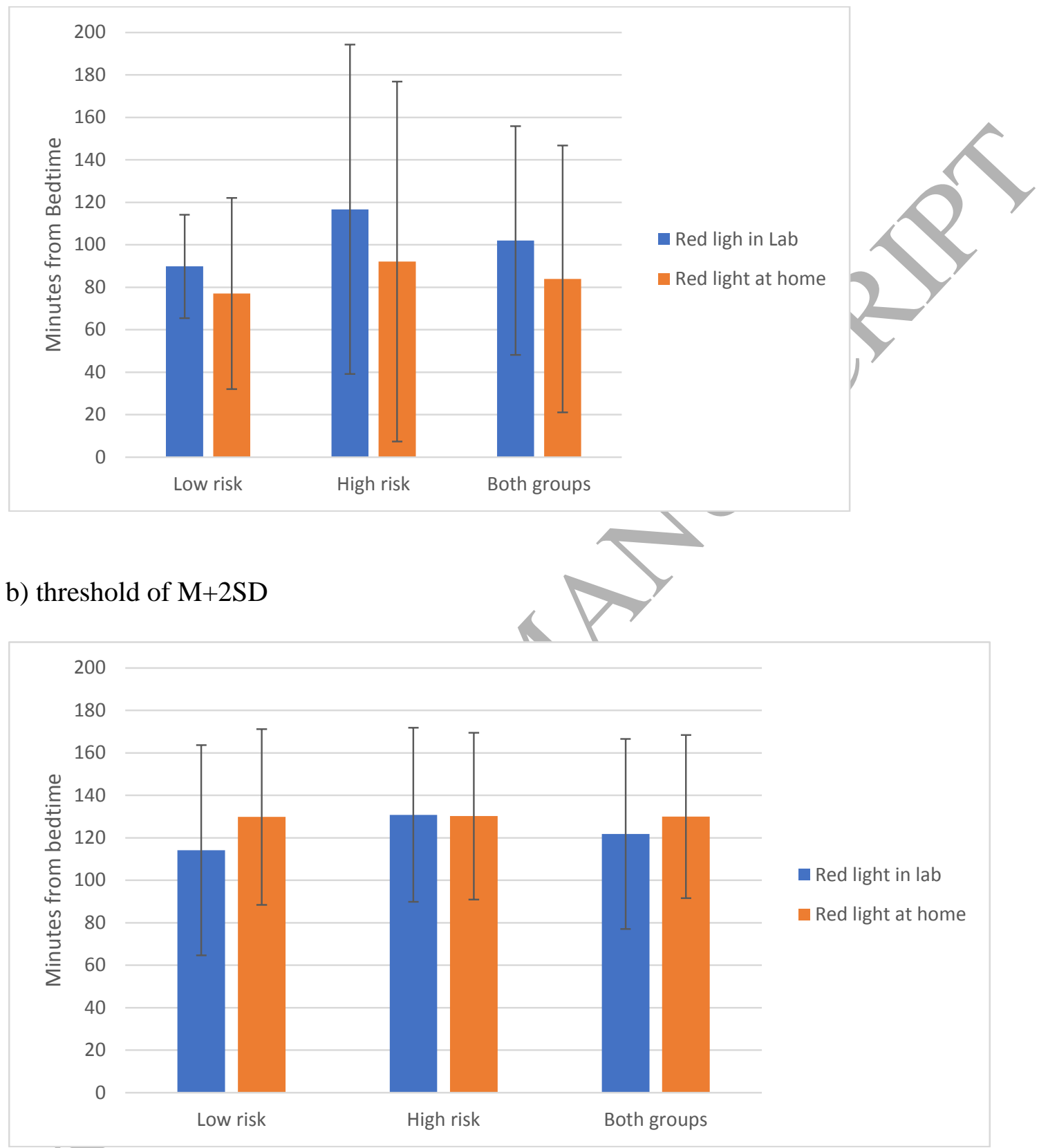